\title{
Butterflies of Amazon and Cerrado remnants of Maranhão, Northeast Brazil
}

\author{
Lucas Pereira Martins ${ }^{* 1,2}$, Elias da Costa Araujo Junior ${ }^{1,3}$, Ananda Regina Pereira Martins ${ }^{1,4}$, \\ Mairla Santos Colins ${ }^{I}$, Gabriela Cristina Fonseca Almeida ${ }^{I} \&$ Gisele Garcia Azevedo $^{I}$ \\ ${ }^{1}$ Universidade Federal do Maranhão, Departamento de Biologia, São Luís, Maranhão, Brazil. \\ ${ }^{2}$ Universidade Federal de Goiás - Programa de Pós-Graduação em Ecologia e Evolução, Goiânia, Goiás, Brazil. \\ ${ }^{3}$ Museu de Zoologia, Universidade de São Paulo, São Paulo, Brazil. \\ ${ }^{4} \mathrm{McGill}$ University, Department of Biology, Montreal, Quebec, Canada. \\ *Corresponding author: Lucas Pereira Martins,e-mail: martinslucas.p@gmail.com
}

MARTINS, L. P., ARAUJO JUNIOR, E. C., MARTINS, A. R. P., COLINS, M. S., ALMEIDA, G. C. F., AZEVEDO, G. G. Butterflies of Amazon and Cerrado remnants of Maranhão, Northeast Brazil. Biota Neotropica. 17(3): e20170335. http://dx.doi.org/10.1590/1676-0611-BN-2017-0335

\begin{abstract}
Species inventories are important tools to evaluate biodiversity losses and contribute to the conservation of endangered areas. The Amazon and Cerrado are the largest Brazilian biomes and represent some of the most threatened regions of the country. Due to its location between these biomes, the state of Maranhão, Northeast Brazil, possesses a great variety of habitats and a high local diversity. Nonetheless, few faunistic inventories of diversified groups have been performed in the state. In the specific case of butterflies, a well-known biological indicator, no inventories have been published in the past years. This study aimed to expand the knowledge on the composition of butterflies in Amazon and Cerrado remnants of Maranhão. Butterflies were sampled between 2011 and 2015 across eight municipalities of the state. Captures were made through entomological nets and baited traps. In total, 189 species were sampled, of which 165 were captured in the Amazon, 65 in the Cerrado and 41 in both biomes. We sampled 167 species through entomological nets and 43 through baited traps, representing 12\% of similarity in species composition between sampling methods. We estimate that the recorded species represent a small subset of the butterflies from Maranhão. Therefore, long-term researches in poorly studied areas of the state are recommended to identify novel and/or endemic taxa.
\end{abstract}

Keywords: tropical forest, diversity, Lepidoptera, Neotropical region, savanna.

\section{Borboletas de remanescentes de Amazônia e Cerrado do Maranhão, nordeste do Brasil}

Resumo: Inventários de espécies são ferramentas importantes para avaliar perdas de biodiversidade e contribuir para a conservação de áreas ameaçadas. A Amazônia e o Cerrado são os maiores biomas brasileiros e representam algumas das regiões mais ameaçadas do país. Devido à sua localização entre estes biomas, o estado do Maranhão, nordeste do Brasil, possui uma grande variedade de habitats e uma alta diversidade local. No entanto, poucos inventários faunísticos de grupos diversificados foram realizados no estado. No caso específico de borboletas, um indicador biológico bem conhecido, nenhum inventário foi publicado nos últimos anos. Este estudo objetivou expandir o conhecimento sobre a composição de borboletas de remanescentes de Amazônia e Cerrado do Maranhão. As borboletas foram amostradas entre 2011 e 2015 em oito municípios do estado. As capturas foram realizadas através de redes entomológicas e armadilhas com iscas. No total, 189 espécies foram amostradas, das quais 165 foram capturadas na Amazônia, 65 no Cerrado e 41 em ambos os biomas. Nós amostramos 167 espécies através de redes entomológicas e 43 através de armadilhas com iscas, representando $12 \%$ de similaridade na composição de espécies entre métodos de amostragem. Nós estimamos que as espécies registradas representam um pequeno subconjunto das borboletas do Maranhão. Deste modo, pesquisas de longa duração em áreas pouco estudadas do estado são recomendadas para identificar taxa novos e/ou endêmicos.

Palavras-chave: floresta tropical, diversidade, Lepidoptera, região Neotropical, savana.

\section{Introduction}

Species inventories contribute to the conservation of endangered areas by providing relevant data for conservation plans, such as occurrence, richness and diversity (Kremen et al. 1993, Santos et al. 2008, Santos et al. 2016). Unfortunately, conservation plans are usually restricted to studies focusing vertebrates and higher plants (Santos et al. 2008). The scarcity of basic knowledge studies on megadiverse groups, such as insects, increases the difficulty of cataloging all species present in a region (Santos et al. 2008). Despite that, insects are suggested as suitable biological indicators in studies of environmental monitoring and evaluations of natural landscape diversity and integrity. Thus, insect 
inventories are important tools to biological evaluations in regions threatened by anthropic disturbances (Kim 1993, Brown Jr 1997, Uehara-Prado et al. 2007, Santos et al. 2016).

Among the insects, butterflies are considered excellent organisms to indicate the "health state" of ecosystems and an effective "umbrella group" for biodiversity conservation (Uehara-Prado et al. 2007, Bonebrake et al. 2010, Santos et al. 2016). There are about 3,300 species of butterflies in Brazil (Brown Jr 1996, Lewinsohn et al. 2005, Francini et al. 2011), but few inventories have been performed in large biomes of the country (Santos et al. 2008). The low number of researchers and difficult access to some sites partially explain the lack of information for many areas of Brazil, especially within the North and Northeast regions (Santos et al. 2008). As a consequence, relatively little is still known about the biodiversity of butterflies in the country considering its vast area, hindering the development of management and conservation strategies (Santos et al. 2008).

The Amazon is the largest biome in Brazil and possesses the world highest absolute rate of forest reduction (Laurance et al. 2000, Silva et al. 2005). Anthropogenic effects caused by the insertion of enterprises and the implementation of monocultures and livestock are the main causes of deforestation, especially in the Eastern portion, which is part of the "deforestation arch" (Silva et al. 2005, Vieira et al. 2008, Martins \& Oliveira 2011). Considering the Amazonian high biodiversity, information on insect composition and distribution remains scarce (Santos et al. 2008). Butterfly inventories have been performed in the Amazon since the $19^{\text {th }}$ century (Bates 1867, Santos et al. 2008), but many of these do not present a delimitation of the sampled area, reducing their scientific reliability (Santos et al. 2008, Casagrande et al. 2012).

The Cerrado is the second largest biome in Brazil and consists of a mosaic of vegetation types, varying from savannas to dense forests (Klink $\&$ Machado 2005). Human occupation has caused extensive habitat loss and transformed large areas of this domain into crops and pastures (Klink
\& Machado 2005, Diniz-Filho et al. 2009). Due to the increasing land-use and the high degree of endemism, the Cerrado is considered a biodiversity hotspot (Myers et al. 2000). However, despite the existence of previous butterfly lists performed in this biome, butterfly fauna is poorly understood especially in regions with insufficient number of research centers, such as the state of Maranhão, one of the poorest known areas in Brazil regarding butterflies (Santos et al. 2008).

The state of Maranhão, Northeast Brazil, contains portions of Amazon, Cerrado and Caatinga vegetation, contributing to the maintenance of a high local diversity. Patterns of habitat reduction are evident within the Amazon and Cerrado areas of Maranhão, and major deforestations are already assumed for the next years in these areas (Vieira et al. 2008, Diniz-Filho et al. 2009, Martins \& Oliveira 2011, Barreto et al. 2012). For this reason, long-term butterfly inventories are crucial for the development and application of efficient conservation measures in the state. The present study aims to broaden the knowledge of the butterfly fauna in the state of Maranhão and provide perspectives for future researches.

\section{Material and Methods}

\section{Study areas}

Field work was performed in forest remnants of Amazon (six municipalities) and Cerrado (two municipalities) (Figure 1). The studied remnants of Amazon forest presented different vegetation types and degrees of human impact (Figure 2): (1) Maracanã, located in the municipality of São Luís ( $2^{\circ} 36^{\prime} \mathrm{S}$ and $\left.44^{\circ} 17^{\prime} \mathrm{W}\right)$, possesses an area of 1,813 ha and vegetation characterized as open ombrophilous forest with high anthropic impacts in several points; (2) Sítio Aguahy, located in the municipality of São José de Ribamar ( $2^{\circ} 38^{\prime} \mathrm{S}$ and $\left.44^{\circ} 08^{\prime} \mathrm{W}\right)$, possesses an area of 635 ha composed by portions of stacional semidecidous forest, being

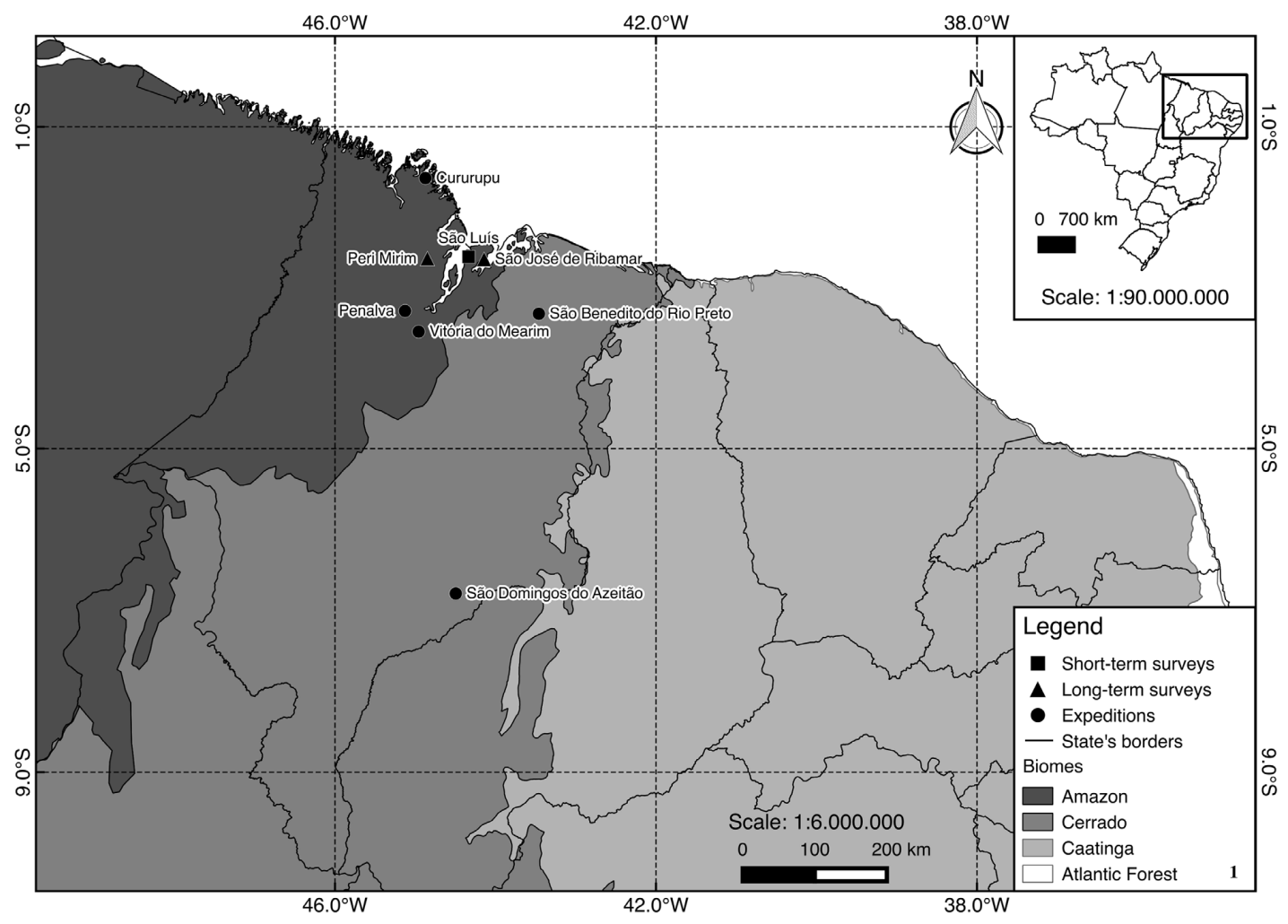

Figure 1. Map of the state of Maranhão, Northeast Brazil. Six study areas were selected in the Amazon and two study areas were selected in the Cerrado. Projection system: UTM. Datum: WGS 84. 

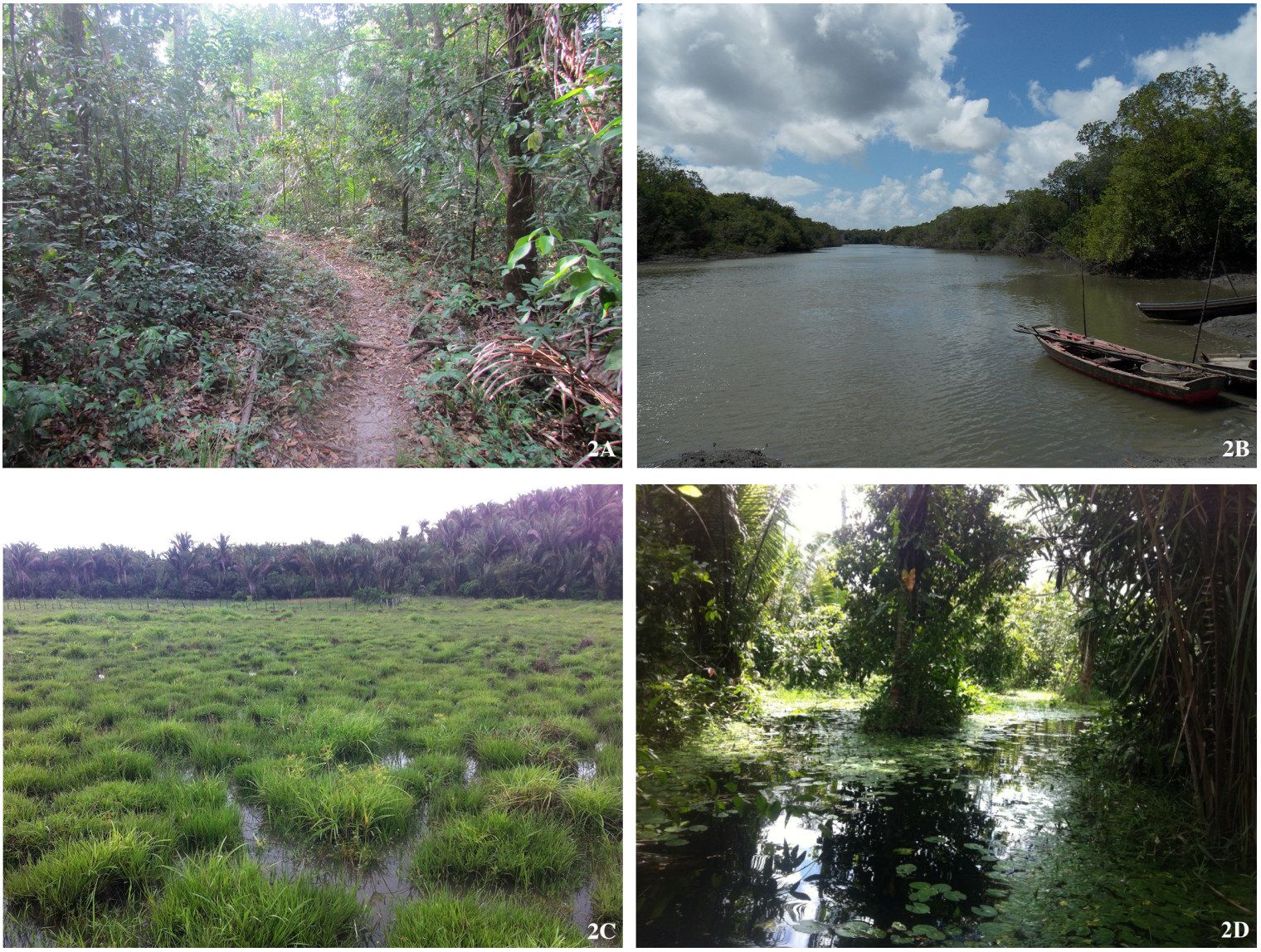

Figure 2. Studied landscapes of the Amazon biome in the state of Maranhão, Northeast Brazil. A. Stacional semideciduous forest in São José de Ribamar. B. Transition zone between Amazon forest and coastal vegetation in Cururupu. C. Natural open fields in Peri Mirim. D. Amazon forest remnant in Vitória do Mearim.

surrounded by crops of Manihot sp. (Euphorbiaceae) and Zea sp. (Poaceae); (3) Cururupu ( $1^{\circ} 49^{\prime} \mathrm{S}$ and $\left.44^{\circ} 51^{\prime} \mathrm{W}\right)$, located in a coastal region inside the Environmental Protection Area of "Reentrâncias Maranhenses", is characterized by the predominance of transition zones between Amazon forest and coastal vegetation, with anthropic impacts of the surrounding villages; (4) Parque Agroecológico de Buritirana, located in Peri Mirim ( $2^{\circ} 38^{\prime} \mathrm{S}$ and $\left.44^{\circ} 50^{\prime} \mathrm{W}\right)$; (5) Fazenda Coque, located in Vitória do Mearim ( $3^{\circ} 32^{\prime} \mathrm{S}$ and $\left.44^{\circ} 57^{\prime} \mathrm{W}\right)$; and (6) Fazenda Canadá \& Boa Esperança, located in Penalva ( $3^{\circ} 17^{\prime} \mathrm{S}$ and $\left.45^{\circ} 07^{\prime} \mathrm{W}\right)$, are situated in the region of "Baixada Maranhense" - 1,775,035 ha of Amazon forest remnants characterized by flooded areas and natural open fields inserted in a matrix of urban settlements and secondary forests.

The study areas of Cerrado were selected in two municipalities (Figure 3): (7) São Benedito do Rio Preto ( $3^{\circ} 19^{\prime} \mathrm{S}$ and $43^{\circ} 31^{\prime} \mathrm{W}$ ), located in the Northeast of Maranhão, is characterized by tablelands and gallery forests following the Preto river; and (8) São Domingos do Azeitão $\left(6^{\circ} 47^{\prime} \mathrm{S}\right.$ and $\left.44^{\circ} 29^{\prime} \mathrm{W}\right)$, located between the headwaters of Itapecuru and Alpercatas rivers, is characterized by open areas with sparse trees and gallery forests, being surrounded by soy crops.

\section{Butterfly sampling and identification}

Butterflies were sampled between 2011 and 2015 by two to four collectors in each locality. Field trips were classified into three categories according to the duration and sampling effort: (1) Short-term survey: two sampling days; (2) Long-term surveys: two sampling days per month during 24 months; and (3) Expeditions: one sampling week.
Using entomological nets, we surveyed for butterflies every sampling day. Butterflies were sampled along transects that varied in extension from $1-5 \mathrm{~km}$. At least two collectors walked along transects collecting with entomological nets in a given amount of time (from $8-12$ a.m. and from $2-5$ p.m., totalling 7 hours*person/day). Cylindrical traps were also used to capture fruit-feeding butterflies (family Nymphalidae) (Uehara-Prado et al. 2007, Freitas et al. 2014). In each transect, at least five traps were disposed with minimum distances of 100 meters between traps. Five traps were used in our short-term survey and 10 traps were used in expeditions. For the long-term surveys, 10 traps were used monthly in Peri Mirim and 18 traps were used monthly in São José de Ribamar. In total, 707 traps were disposed in the Amazon biome and 20 in the Cerrado. Traps were placed 1.5 meters from the ground and baited with cat feces, rotting fish or fruits. The proportion of four traps baited with bananas or mango with sugarcane juice to one trap baited with cat feces or rotting fish was maintained for all transects in expeditions. On the other hand, only bananas with sugarcane juice were used as baits in short and long-term surveys. We inspected traps twice a day. For each collected specimen, date, time and habitat type was recorded.

Captured butterflies were taken to the Laboratório de Ecologia e Sistemática de Insetos Polinizadores e Predadores (LESPP/UFMA) and identified through comparison with specimens from the Museu de Zoologia da Universidade de São Paulo (MZUSP), Museu de Zoologia da Universidade Estadual de Campinas (ZUEC), Coleção Entomológica Pe. Jesus Santiago Moure (DZUP) and specialized catalogues (D'abrera 1995, Garwood et al. 2009). Taxonomy follows mostly Lamas (2004), Mielke 

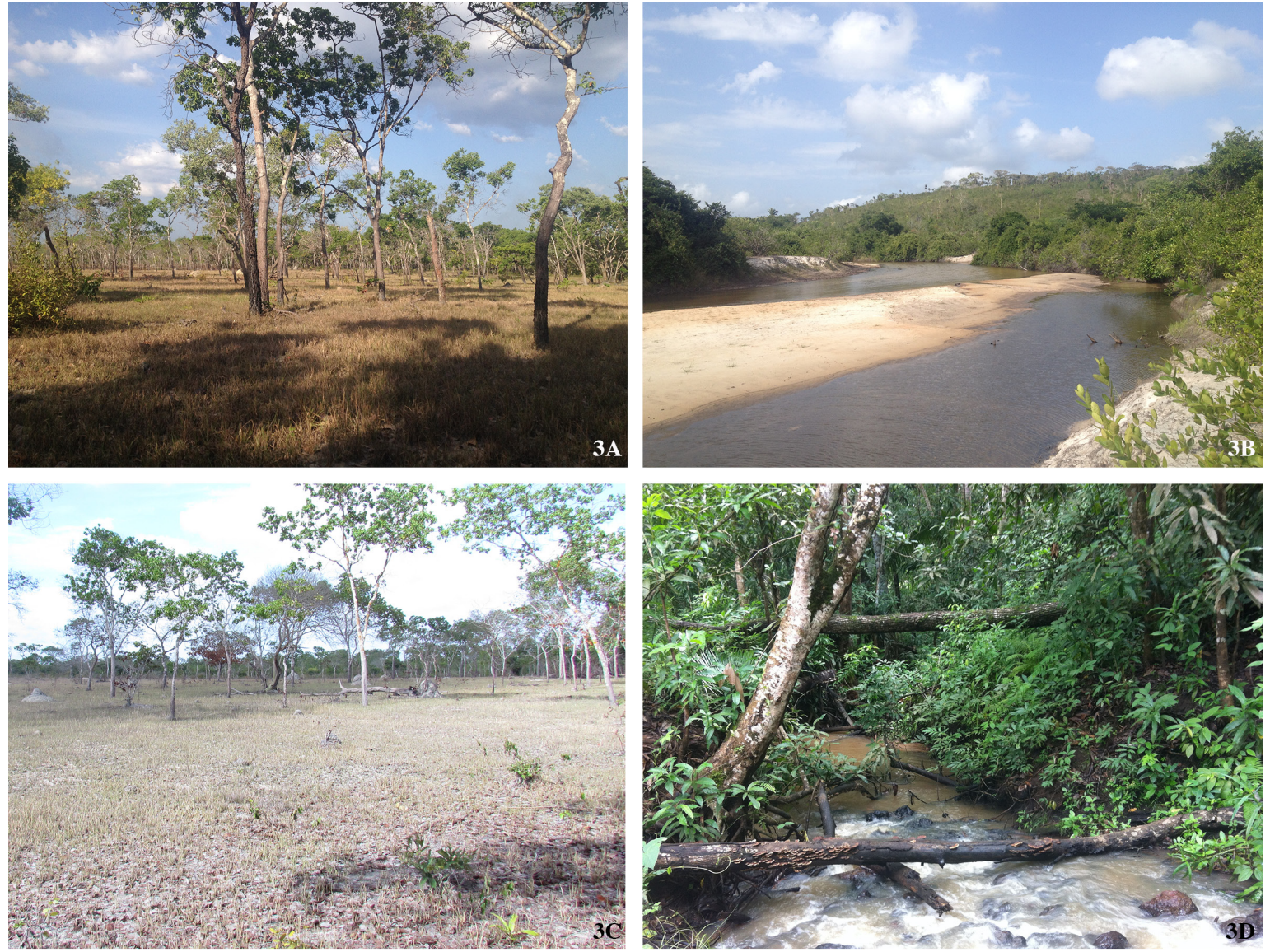

Figure 3. Studied landscapes of the Cerrado biome in the state of Maranhão, Northeast Brazil. A. Tablelands of Cerrado in the Northeast of the state. B. The Preto river and its gallery forest. C. Typical Cerrado vegetation in São Benedito do Rio Preto. D. Gallery forest following the Alpercatas river in São Domingos do Azeitão.

(2005) and Duarte \& Robbins (2010). Vouchers were deposited in four collections: LESPP/UFMA, MZUSP, ZUEC and DZUP.

\section{Data analysis}

We used the Jaccard index $(\mathrm{J})$ to evaluate quantitatively the similarity in species composition between sampling methods (entomological nets and baited traps). This index is based on presence-absence data and has been widely used in ecological studies (Magurran 2004, Jost et al. 2011). In order to compare our list to other lists performed in the state, we searched for papers in the Web of Science, Scopus and Google Scholar databases. Only papers that provided a list of butterfly species and a clear indication that the field work was performed in the state of Maranhão (e.g.: coordinates, region or municipality) were included in the analysis.

\section{Results}

We recorded 189 butterfly species, of which 165 were captured in the Amazon, 65 in the Cerrado and 41 in both biomes. Nymphalidae was the best represented family, with $41.3 \%$ of the total richness $(n=78)$, followed by Hesperiidae $(n=53)$, Riodinidae $(n=29)$, Lycaenidae $(n=16)$, Pieridae $(\mathrm{n}=10)$ and Papilionidae $(\mathrm{n}=3)$ (Table 1). The richest subfamilies were, respectively, Riodininae with 29 species ( $15.3 \%$ of the species collected), Satyrinae with 24 species $(12.7 \%)$ and Hesperiinae with 23 species (12.2\%). Hamadryas februa (Hübner, [1823]), an indicator of disturbed habitats (Brown Jr 1992), was the most widespread species, occurring in all municipalities. Regarding sampling effort, 146 species were captured in long-term surveys, 112 in expeditions and 15 in our short-term survey.
Specifically, the municipality of Peri Mirim presented the greatest number of species $(\mathrm{n}=101)$, followed by São José de Ribamar $(\mathrm{n}=99)$. A total of 167 species were sampled through entomological nets and 43 through baited traps. Similarity in species composition between sampling methods was recorded in $12 \%(\mathrm{~J}=0.12)$.

Four lists performed in the state of Maranhão that meet the established criteria were included in the analyzes (Table 2). Of these studies, three used exclusively entomological nets to collect butterflies (Bates 1867, Garcia et al. 1990, Garcia \& Bergman 1994), and one used cylindrical traps baited with bananas and sugarcane juice (Ramos 2000). Additionally, all these studies were performed in the Amazon biome, being two of these at the state capital, São Luís (Garcia et al. 1990, Garcia \& Bergman 1994).

\section{Discussion}

The butterfly fauna captured in this study represents approximately $6 \%$ of the species recorded for Brazil (Brown Jr 1996, Lewinsohn et al. 2005, Francini et al. 2011). It is known that species richness is strongly dependent on sampling effort, partially explaining the lowest richness in our short-term survey when compared to our expeditions and long-term surveys. The influence of sampling effort on species richness is also observed when we compare our total richness ( 189 species) to intensively sampled areas in the Amazon, such as Rondônia (843) (Emmel \& Austin 1990), Parque Nacional del Manu, in Peru (1,300) (Robbins et al. 1996) and Parque Estadual do Chandless, Acre (482) (Mielke et al. 2010), and Cerrado sites, such as Distrito Federal (504) (Emery et al. 2006). Comparing our results with those from other biomes, such as the Atlantic forest, one of the most 
Table 1. List of butterflies of Amazon and Cerrado remnants of the state of Maranhão, Northeast Brazil.

\begin{tabular}{|c|c|c|c|c|c|c|c|c|c|c|}
\hline \multirow{2}{*}{ Taxa (Family, Subfamily, Species, Subspecies) } & \multicolumn{6}{|c|}{ Amazon } & \multicolumn{2}{|c|}{ Cerrado } & \multicolumn{2}{|c|}{ Sampling method } \\
\hline & $\mathbf{C P}$ & PV & PM & SJR & SL & VT & SBR & SDA & $\mathbf{E N}$ & BT \\
\hline \multicolumn{11}{|l|}{ Nymphalidae $(S=78)$} \\
\hline \multicolumn{11}{|l|}{ Biblidinae $(S=14)$} \\
\hline Biblis hyperia (Cramer, 1779) & & & & $\mathrm{x}$ & & & & & & $\mathrm{x}$ \\
\hline Callicore astarte astarte (Cramer 1779) & & & & $\mathrm{x}$ & & & & & & $\mathrm{x}$ \\
\hline Catonephele acontius (Linnaeus 1771) & & & & $\mathrm{x}$ & & & & & & $\mathrm{x}$ \\
\hline Dynamine agacles (Dalman, 1823) & $\mathrm{x}$ & & & & & & & & $\mathrm{x}$ & \\
\hline Dynamine myrson myrson (Doubleday, 1849) & $\mathrm{x}$ & & & & & & & & $\mathrm{x}$ & \\
\hline Dynamine paulina (Bates, 1865) & & & & $\mathrm{x}$ & & & & & $\mathrm{x}$ & \\
\hline Dynamine postverta postverta (Cramer, 1779) & & & $\mathrm{x}$ & $\mathrm{x}$ & & & & & $\mathrm{x}$ & \\
\hline Ectima iona E. Doubleday, [1848] & $\mathrm{x}$ & & & & & & & & & $\mathrm{x}$ \\
\hline Eunica maja (Fabricius, 1775) & & & & $\mathrm{x}$ & & & & & & $\mathrm{x}$ \\
\hline Hamadryas amphinome (Linnaeus, 1767) & $\mathrm{x}$ & & $\mathrm{x}$ & $\mathrm{x}$ & & $\mathrm{x}$ & $\mathrm{x}$ & & $\mathrm{x}$ & $\mathrm{x}$ \\
\hline Hamadryas chloe (Stoll, 1787) & $\mathrm{x}$ & & $\mathrm{x}$ & $\mathrm{x}$ & & & $\mathrm{x}$ & & $\mathrm{x}$ & $\mathrm{x}$ \\
\hline Hamadryas februa (Hübner, [1823]) & $\mathrm{x}$ & $\mathrm{x}$ & $\mathrm{x}$ & $\mathrm{x}$ & $\mathrm{x}$ & $\mathrm{x}$ & $\mathrm{x}$ & $\mathrm{x}$ & $\mathrm{x}$ & $\mathrm{x}$ \\
\hline Hamadryas feronia (Linnaeus, 1758) & & & $\mathrm{x}$ & $\mathrm{x}$ & $\mathrm{x}$ & $\mathrm{x}$ & $\mathrm{x}$ & $\mathrm{x}$ & $\mathrm{x}$ & $\mathrm{x}$ \\
\hline Hamadryas laodamia (Cramer, 1777) & & & & $\mathrm{x}$ & & & & & & $\mathrm{x}$ \\
\hline \multicolumn{11}{|l|}{ Charaxinae $(S=10)$} \\
\hline Archaeoprepona demophon (Linnaeus, 1758) & & & $\mathrm{x}$ & $\mathrm{x}$ & $\mathrm{x}$ & $\mathrm{x}$ & & & & $\mathrm{x}$ \\
\hline Archaeoprepona demophoon (Hübner, [1814]) & & & $\mathrm{x}$ & & & & & & & $\mathrm{x}$ \\
\hline Fountainea ryphea (Cramer, 1775) & & & $\mathrm{x}$ & $\mathrm{x}$ & $\mathrm{x}$ & $\mathrm{x}$ & & & $\mathrm{x}$ & $\mathrm{x}$ \\
\hline Hypna clytemnestra (Cramer, 1777) & & & $\mathrm{x}$ & $\mathrm{x}$ & & & & & $\mathrm{x}$ & $\mathrm{x}$ \\
\hline Memphis acidalia (Hübner, [1819]) & & & & $\mathrm{x}$ & & & & & & $x$ \\
\hline Memphis leonida (Stoll, 1782) & & & & $\mathrm{x}$ & & & & & & $\mathrm{x}$ \\
\hline Prepona laertes (Hübner, [1811]) & & & $\mathrm{x}$ & $\mathrm{x}$ & & $\mathrm{x}$ & & & & $\mathrm{x}$ \\
\hline Prepona pheridamas (Cramer, 1777) & & & $\mathrm{x}$ & $\mathrm{x}$ & & & & & $\mathrm{x}$ & $\mathrm{x}$ \\
\hline Prepona pseudomphale Le Moult, 1932 & & & $\mathrm{x}$ & & & & & & & $\mathrm{x}$ \\
\hline Zaretis isidora (Cramer, 1779) & & $\mathrm{x}$ & $\mathrm{x}$ & $\mathrm{x}$ & & $\mathrm{x}$ & $\mathrm{x}$ & & $\mathrm{x}$ & $\mathrm{x}$ \\
\hline \multicolumn{11}{|l|}{ Cyrestinae $(S=2)$} \\
\hline Marpesia chiron (Fabricius, 1775) & & & $\mathrm{x}$ & $\mathrm{x}$ & & & & & $\mathrm{x}$ & \\
\hline Marpesia petreus (Cramer, 1776) & & & $\mathrm{x}$ & $\mathrm{x}$ & & $\mathrm{x}$ & & & $\mathrm{x}$ & \\
\hline \multicolumn{11}{|l|}{ Danainae $(S=5)$} \\
\hline Danaus eresimus (Cramer, 1777) & & & $\mathrm{x}$ & & & & & & $\mathrm{x}$ & \\
\hline Danaus gilippus (Cramer, 1775) & & & $\mathrm{x}$ & & & & & $\mathrm{x}$ & $\mathrm{x}$ & \\
\hline Danaus sp Kluk, 1780 & & & $\mathrm{x}$ & & & & & & $\mathrm{x}$ & \\
\hline Lycorea halia (Hübner, 1816) & & & $\mathrm{x}$ & $\mathrm{x}$ & & $\mathrm{x}$ & & & $\mathrm{x}$ & \\
\hline Methona sp Doubleday, 1847 & & & $\mathrm{x}$ & $\mathrm{x}$ & $\mathrm{x}$ & & & & $\mathrm{x}$ & \\
\hline \multicolumn{11}{|l|}{ Heliconiinae ( $\mathrm{S}=13)$} \\
\hline Agraulis vanillae (Linnaeus, 1758) & & & $\mathrm{x}$ & & & & & & $\mathrm{x}$ & \\
\hline Dryas iulia (Fabricius, 1775) & $\mathrm{x}$ & & $\mathrm{x}$ & $\mathrm{x}$ & $\mathrm{x}$ & & & $\mathrm{x}$ & $\mathrm{x}$ & \\
\hline Dryadula phaetusa (Linnaeus, 1758) & & & $\mathrm{x}$ & $\mathrm{x}$ & & $\mathrm{x}$ & & & $\mathrm{x}$ & \\
\hline Euptoieta hegesia (Cramer, 1779) & & & $\mathrm{x}$ & $\mathrm{X}$ & & $\mathrm{x}$ & $\mathrm{x}$ & & $\mathrm{x}$ & \\
\hline Heliconius antiochus (Linnaeus, 1767) & $\mathrm{x}$ & $\mathrm{x}$ & $\mathrm{x}$ & $\mathrm{x}$ & & & $\mathrm{x}$ & & $\mathrm{x}$ & \\
\hline Heliconius erato phyllis (Fabricius, 1775) & & & & $\mathrm{x}$ & & & & & $\mathrm{x}$ & \\
\hline Heliconius melpomene melpomene (Linnaeus, 1758) & $\mathrm{x}$ & $\mathrm{x}$ & $\mathrm{x}$ & $\mathrm{x}$ & & $\mathrm{x}$ & & & $\mathrm{x}$ & \\
\hline Heliconius melpomene nanna Stichel, 1899 & & & $\mathrm{x}$ & $\mathrm{x}$ & $\mathrm{x}$ & & & $\mathrm{x}$ & $\mathrm{x}$ & \\
\hline Heliconius ricini (Linnaeus, 1758) & & & $\mathrm{x}$ & $\mathrm{x}$ & & $\mathrm{x}$ & & & $\mathrm{x}$ & \\
\hline Heliconius numata (Cramer, 1780) & & & $\mathrm{x}$ & $\mathrm{x}$ & & & & & $\mathrm{x}$ & \\
\hline Heliconius sara sara (Fabricius, 1793) & & & & $\mathrm{X}$ & & & & $\mathrm{x}$ & $\mathrm{x}$ & \\
\hline Neruda mentis Moreira \& Mielke, 2010 & & & & & & & & $\mathrm{x}$ & $\mathrm{x}$ & \\
\hline Philaethria dido (Linnaeus, 1763) & $\mathrm{x}$ & & $\mathrm{x}$ & $\mathrm{x}$ & & $\mathrm{x}$ & & & $\mathrm{x}$ & \\
\hline \multicolumn{11}{|l|}{ Limenitidinae $(S=2)$} \\
\hline Adelpha cytherea cytherea (Linnaeus, 1758) & $\mathrm{x}$ & & & $\mathrm{x}$ & & & & & $\mathrm{x}$ & \\
\hline Adelpha iphiclus iphiclus (Linnaeus, 1758) & $\mathrm{x}$ & & $\mathrm{x}$ & $\mathrm{x}$ & & $\mathrm{x}$ & & & $\mathrm{x}$ & \\
\hline \multicolumn{11}{|l|}{ Nymphalinae $(S=8)$} \\
\hline Anartia amathea (Linnaeus, 1758) & & & $\mathrm{x}$ & & & & & $\mathrm{x}$ & $\mathrm{x}$ & \\
\hline Anartia jatrophae (Linnaeus, 1763) & $\mathrm{x}$ & & $\mathrm{x}$ & $\mathrm{x}$ & & $\mathrm{x}$ & & $\mathrm{x}$ & $\mathrm{x}$ & \\
\hline
\end{tabular}

$\mathrm{CP}$ = Cururupu; PV = Penalva; PM = Peri Mirim; SJR = São José de Ribamar; SL = São Luís; VT = Vitória do Mearim; SBR = São Benedito do Rio Preto; SDA = São Domingos do Azeitão; EN = Entomological net; BT = Baited trap. 
Table 1. Continued...

\begin{tabular}{|c|c|c|c|c|c|c|c|c|c|c|}
\hline \multirow{2}{*}{ Taxa (Family, Subfamily, Species, Subspecies) } & \multicolumn{6}{|c|}{ Amazon } & \multicolumn{2}{|c|}{ Cerrado } & \multicolumn{2}{|c|}{ Sampling method } \\
\hline & $\mathbf{C P}$ & PV & $\mathbf{P M}$ & SJR & SL & VT & SBR & SDA & $\mathbf{E N}$ & BT \\
\hline Colobura dirce (Linnaeus, 1758) & $\mathrm{x}$ & & $\mathrm{x}$ & $\mathrm{x}$ & & $\mathrm{x}$ & & & $\mathrm{x}$ & $\mathrm{x}$ \\
\hline Historis acheronta (Fabricius, 1775) & & & $\mathrm{x}$ & $\mathrm{x}$ & & & & & & $\mathrm{x}$ \\
\hline Historis odius (Fabricius, 1775) & & & $\mathrm{x}$ & $\mathrm{x}$ & & & & & & $\mathrm{x}$ \\
\hline Junonia evarete (Cramer, 1779) & $\mathrm{x}$ & $\mathrm{x}$ & $\mathrm{x}$ & $\mathrm{x}$ & & $\mathrm{x}$ & & & $\mathrm{x}$ & $\mathrm{x}$ \\
\hline Siproeta stelenes (Linnaeus, 1758) & & & $\mathrm{x}$ & $\mathrm{x}$ & & & & & $\mathrm{x}$ & \\
\hline Tigridia acesta (Linnaeus, 1758) & $\mathrm{x}$ & & & & & & & & $\mathrm{x}$ & \\
\hline \multicolumn{11}{|l|}{ Satyrinae $(S=24)$} \\
\hline Brassolis sophorae (Linnaeus, 1758) & & & $\mathrm{x}$ & & & & & $\mathrm{x}$ & $\mathrm{x}$ & \\
\hline Catoblepia berecynthia (Cramer, 1777) & $\mathrm{x}$ & & $\mathrm{x}$ & $\mathrm{x}$ & & & $\mathrm{x}$ & $\mathrm{x}$ & $\mathrm{x}$ & $\mathrm{x}$ \\
\hline Cissia penelope (Fabricius, 1775) & $\mathrm{x}$ & $\mathrm{x}$ & & $\mathrm{x}$ & & $\mathrm{x}$ & & $\mathrm{x}$ & $\mathrm{x}$ & $\mathrm{x}$ \\
\hline Caligo teucer (Linnaeus, 1758) & & & $\mathrm{x}$ & $\mathrm{x}$ & & & & & & $\mathrm{x}$ \\
\hline Caligo illioneus (Cramer, 1775) & & & $\mathrm{x}$ & & $\mathrm{x}$ & $\mathrm{x}$ & & & $\mathrm{x}$ & $\mathrm{x}$ \\
\hline Hermeuptychia hermes (Fabricius, 1775) & & & & $\mathrm{x}$ & & $\mathrm{x}$ & & $\mathrm{x}$ & $\mathrm{x}$ & $\mathrm{x}$ \\
\hline Magneuptychia libye (Linnaeus, 1767) & & & $\mathrm{x}$ & $\mathrm{x}$ & & $\mathrm{x}$ & & & $\mathrm{x}$ & $\mathrm{x}$ \\
\hline Magneuptychia ocypete (Fabricius, 1776) & & & & $\mathrm{x}$ & & & & & & $\mathrm{x}$ \\
\hline Magneuptychia pallema (Schaus, 1902) & & & & $\mathrm{x}$ & & & & & & $\mathrm{x}$ \\
\hline Morpho helenor (Cramer, 1776) & $\mathrm{x}$ & & $\mathrm{x}$ & $\mathrm{x}$ & & $\mathrm{x}$ & & & $\mathrm{x}$ & $\mathrm{x}$ \\
\hline Morpho menelaus terrestris (Butler, 1866) & & & & $\mathrm{x}$ & $\mathrm{x}$ & & & & $\mathrm{x}$ & $\mathrm{x}$ \\
\hline Morpho rhetenor Butler, 1866 & & & & $\mathrm{x}$ & & & & & $\mathrm{x}$ & $\mathrm{x}$ \\
\hline Narope panniculus Stichel, 1904 & & & & & & & $\mathrm{x}$ & & & $\mathrm{x}$ \\
\hline Opsiphanes invirae (Hübner, [1808]) & & & $\mathrm{x}$ & $\mathrm{x}$ & & & & & $\mathrm{x}$ & $\mathrm{x}$ \\
\hline Opsiphanes quiteria (Stoll, 1780) & & & & $\mathrm{x}$ & & $\mathrm{x}$ & & & $\mathrm{x}$ & $\mathrm{x}$ \\
\hline Pierella hyalinus (Gmelin, [1790]) & $\mathrm{x}$ & & & $\mathrm{x}$ & & & & & $\mathrm{x}$ & \\
\hline Pierella lamia (Sulzer, 1776) & & & & $\mathrm{x}$ & & & & $\mathrm{x}$ & $\mathrm{x}$ & \\
\hline Pharneuptychia innocentia (C. Felder \& R. Felder, 1867) & & & & & & & $\mathrm{x}$ & & $\mathrm{x}$ & \\
\hline Selenophanes cassiope (Cramer, 1775) & & & & & & & & $\mathrm{x}$ & $\mathrm{x}$ & \\
\hline Taygetis echo (Cramer, 1775) & & & $\mathrm{x}$ & & & & & & & $\mathrm{x}$ \\
\hline Taygetis laches Fabricius, 1793 & & & $\mathrm{x}$ & $\mathrm{x}$ & & & & & $\mathrm{x}$ & $\mathrm{x}$ \\
\hline Taeygetis sosis Hopffer, 1874 & & & $\mathrm{x}$ & & & & & & & $\mathrm{x}$ \\
\hline Yphthimoides renata $($ Stoll, 1780$)$ & & & & $\mathrm{x}$ & & & & & & $\mathrm{x}$ \\
\hline Yphthimoides affinis (Butler, 1867) & & & & & & $\mathrm{x}$ & & & & $\mathrm{x}$ \\
\hline \multicolumn{11}{|l|}{ Lycaenidae $(S=16)$} \\
\hline \multicolumn{11}{|l|}{ Polyommatinae $(\mathrm{S}=2)$} \\
\hline Hemiargus hanno hanno (Stoll, 1790) & & & $\mathrm{x}$ & $\mathrm{x}$ & & & $\mathrm{x}$ & $\mathrm{x}$ & $\mathrm{x}$ & \\
\hline Leptotes cassius (Cramer, 1775) & & & & & & & $\mathrm{x}$ & $\mathrm{x}$ & $\mathrm{x}$ & \\
\hline \multicolumn{11}{|l|}{ Theclinae $(S=14)$} \\
\hline Arawacus aetolus (Sulzer, 1776) & $\mathrm{x}$ & & & $\mathrm{x}$ & & & & $\mathrm{x}$ & $\mathrm{x}$ & \\
\hline Calycopis demonassa (Hewitson, 1868) & & & & $\mathrm{x}$ & & & & & $\mathrm{x}$ & \\
\hline Chlorostrymon telea (Hewitson, 1868) & & & & & & & $\mathrm{x}$ & & $\mathrm{x}$ & \\
\hline Evenus satyroides (Hewitson,1865) & & & & $\mathrm{x}$ & & & & & $\mathrm{x}$ & \\
\hline Iaspis castitas (H. H. Druce, 1907) & & & $\mathrm{x}$ & & & & & & $\mathrm{x}$ & \\
\hline Rekoa palegon (Cramer, 1780) & & & & & & & $\mathrm{x}$ & & $\mathrm{x}$ & \\
\hline Ministrymon megacles (Stoll, 1780) & & & $\mathrm{x}$ & & & & $\mathrm{x}$ & & $\mathrm{x}$ & \\
\hline Ministrymon zilda (Hewitson, 1873) & & & $\mathrm{x}$ & & & & $\mathrm{x}$ & & $\mathrm{x}$ & \\
\hline Nicolaea socia (Hewitson, 1868) & & & & & & & $\mathrm{x}$ & & $\mathrm{x}$ & \\
\hline Panthiades phaleros (Linnaeus, 1767) & & & $\mathrm{x}$ & $\mathrm{x}$ & & & & & $\mathrm{x}$ & \\
\hline Pseudolycaena marsyas (Linnaeus, 1758) & $\mathrm{x}$ & & $\mathrm{x}$ & $\mathrm{x}$ & & & $\mathrm{x}$ & & $\mathrm{x}$ & \\
\hline Strymon mulucha (Hewitson, 1867) & & & $\mathrm{x}$ & & & & $\mathrm{x}$ & & $\mathrm{x}$ & \\
\hline Tmolus echion (Linnaeus, 1767) & & & & & & & $\mathrm{x}$ & & $\mathrm{x}$ & \\
\hline Ziegleria hesperitis (Butler \& H. Druce, 1872) & & & $\mathrm{x}$ & & & & & & $\mathrm{x}$ & \\
\hline \multicolumn{11}{|l|}{ Pieridae $(S=10)$} \\
\hline \multicolumn{11}{|l|}{ Coliadinae $(S=8)$} \\
\hline Anteos menippe (Hübner, [1818]) & $\mathrm{x}$ & & & & & & & & $\mathrm{x}$ & \\
\hline Aphrissa statira statira (Cramer, 1777) & & & $\mathrm{x}$ & $\mathrm{x}$ & & & $\mathrm{x}$ & & $\mathrm{x}$ & \\
\hline Eurema albula (Cramer, 1775) & & & $\mathrm{x}$ & $\mathrm{x}$ & & & $\mathrm{x}$ & & $\mathrm{x}$ & \\
\hline
\end{tabular}

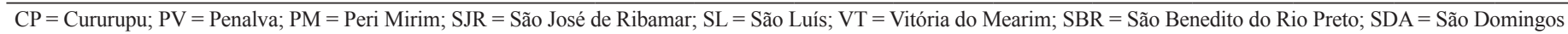
do Azeitão; EN = Entomological net; $\mathrm{BT}=$ Baited trap. 
Table 1. Continued...

\begin{tabular}{|c|c|c|c|c|c|c|c|c|c|c|}
\hline \multirow{2}{*}{ Taxa (Family, Subfamily, Species, Subspecies) } & \multicolumn{6}{|c|}{ Amazon } & \multicolumn{2}{|c|}{ Cerrado } & \multicolumn{2}{|c|}{ Sampling method } \\
\hline & $\mathbf{C P}$ & PV & PM & SJR & SL & VT & SBR & SDA & EN & BT \\
\hline Eurema elathea (Cramer, 1777) & & & & $\mathrm{x}$ & & $\mathrm{x}$ & $\mathrm{x}$ & $\mathrm{x}$ & $\mathrm{x}$ & \\
\hline Phoebis argante argante (Fabricius, 1775) & & & $\mathrm{x}$ & & & $\mathrm{x}$ & & & $\mathrm{x}$ & \\
\hline Phoebis philea philea (Linnaeus, 1763) & & & $\mathrm{x}$ & $\mathrm{x}$ & & & & & $\mathrm{x}$ & \\
\hline Phoebis sennae marcellina (Cramer, 1777) & & & $\mathrm{x}$ & $\mathrm{x}$ & $\mathrm{X}$ & $\mathrm{x}$ & $\mathrm{x}$ & $\mathrm{x}$ & $\mathrm{x}$ & \\
\hline Pyrisitia nise (Cramer, 1775) & & & & $\mathrm{x}$ & & & $\mathrm{x}$ & $\mathrm{x}$ & $\mathrm{x}$ & \\
\hline \multicolumn{11}{|l|}{ Pierinae $(S=2)$} \\
\hline Ascia monuste (Linnaeus, 1764) & & $\mathrm{x}$ & $\mathrm{x}$ & $x$ & & $\mathrm{x}$ & $\mathrm{x}$ & & $\mathrm{x}$ & \\
\hline Itaballia demophile (Linnaeus, 1763) & & & $\mathrm{x}$ & & & $\mathrm{x}$ & & & $\mathrm{x}$ & \\
\hline \multicolumn{11}{|l|}{ Papilionidae $(S=3)$} \\
\hline \multicolumn{11}{|l|}{ Papilioninae $(S=3)$} \\
\hline Battus polydamas (Linnaeus, 1758) & $\mathrm{x}$ & $\mathrm{x}$ & $\mathrm{x}$ & $\mathrm{x}$ & & $\mathrm{x}$ & $x$ & $\mathrm{x}$ & $\mathrm{x}$ & \\
\hline Heraclides thoas (Linnaeus, 1771) & & $\mathrm{x}$ & & $\mathrm{x}$ & & & & $\mathrm{x}$ & $\mathrm{x}$ & \\
\hline Heraclides anchisiades (Esper, 1788) & & & $\mathrm{x}$ & & & & & & $\mathrm{x}$ & \\
\hline \multicolumn{11}{|l|}{ Riodinidae $(S=29)$} \\
\hline \multicolumn{11}{|l|}{ Riodininae ( $S=29)$} \\
\hline Aricoris campestris (Bates, 1868) & & & $\mathrm{x}$ & & & & & $\mathrm{x}$ & $\mathrm{x}$ & \\
\hline Aricoris propitia (Stichel, 1910) & & & $\mathrm{x}$ & & & & & $\mathrm{x}$ & $\mathrm{x}$ & \\
\hline Baeotis euprepes (Bates, 1868) & & & & & & & $\mathrm{x}$ & & $\mathrm{x}$ & \\
\hline Calospila lucianus (Fabricius, 1793) & & & & & & & $\mathrm{x}$ & & $\mathrm{x}$ & \\
\hline Calospila sp Geyer, 1832 & & & $\mathrm{x}$ & $\mathrm{x}$ & & & & $\mathrm{x}$ & $\mathrm{x}$ & \\
\hline Detritivora zama (Bates, 1868) & $\mathrm{x}$ & & & & & & & & $\mathrm{x}$ & \\
\hline Emesis diogenia Prittwitz, 1865 & & & & & & & $\mathrm{x}$ & & $\mathrm{x}$ & \\
\hline Eurybia elvina Stichel, 1910 & & & & $\mathrm{x}$ & & & & & $\mathrm{x}$ & \\
\hline Eurybia patrona Weymer, 1875 & & & & $\mathrm{x}$ & & & & & $\mathrm{x}$ & \\
\hline Helicopis cupido (Linnaeus, 1758) & & & & & & $\mathrm{x}$ & $\mathrm{x}$ & & $\mathrm{x}$ & \\
\hline Isapis agyrtus (Cramer, 1777) & & & & & & & $\mathrm{x}$ & & $\mathrm{x}$ & \\
\hline Juditha odites odites (Cramer, 1775) & & & & & & & $\mathrm{x}$ & & $\mathrm{x}$ & \\
\hline Lemonias zygia Hübner, 1807 & & & $\mathrm{x}$ & $\mathrm{x}$ & & & $\mathrm{x}$ & & $\mathrm{x}$ & \\
\hline Melanis smithiae (Westwood, 1851) & & & & & & & & $\mathrm{x}$ & $\mathrm{x}$ & \\
\hline Mesosemia steli Hewitson 1858 & & & $\mathrm{x}$ & & & & & & $\mathrm{x}$ & \\
\hline Mesosemia sp Hübner, [1819] & & & & $\mathrm{x}$ & & & & & $\mathrm{x}$ & \\
\hline Napaea beltiana beltiana (Bates, 1867) & & & $\mathrm{x}$ & $\mathrm{x}$ & & & & & $\mathrm{x}$ & \\
\hline Napaea eucharila (Bates, 1867) & & & $\mathrm{x}$ & & & & & & $\mathrm{x}$ & \\
\hline Mesene phareus (Cramer, 1777) & $\mathrm{x}$ & & & & & & & & $\mathrm{x}$ & \\
\hline Phaenochitonia cingulus (Stoll, 1790) & & & $\mathrm{x}$ & $\mathrm{x}$ & & & & & $\mathrm{x}$ & \\
\hline Stalachtis phlegia (Cramer, 1779) & & & $\mathrm{x}$ & $\mathrm{x}$ & $\mathrm{x}$ & & $\mathrm{x}$ & & $\mathrm{x}$ & \\
\hline Synargis axenus axenus (Hewitson, 1876) & & & & & & & $\mathrm{x}$ & & $\mathrm{x}$ & \\
\hline Synargis agle (Hewitson, [1853]) & & & $\mathrm{x}$ & & & & & & $\mathrm{x}$ & \\
\hline Synargis calyce (C. Felder \& R. Felder, 1862) & & & $\mathrm{x}$ & & & & $\mathrm{x}$ & & $\mathrm{x}$ & \\
\hline Synargis galena (Bates, 1868) & $\mathrm{x}$ & & & & & & & & $\mathrm{x}$ & \\
\hline Synargis gela (Hewitson, [1853]) & $\mathrm{x}$ & & & & & & & & $\mathrm{x}$ & \\
\hline Thisbe irenea (Stoll, 1780) & $\mathrm{x}$ & & & & & & & & $\mathrm{x}$ & \\
\hline Thisbe molela (Hewitson, 1865) & $\mathrm{x}$ & & & & & & & & $\mathrm{x}$ & \\
\hline Theope foliorum Bates, 1868 & & & & & & & $\mathrm{x}$ & & $\mathrm{x}$ & \\
\hline \multicolumn{11}{|l|}{ Hesperiidae ( $\mathrm{S}=53)$} \\
\hline \multicolumn{11}{|l|}{ Eudaminae $(S=16)$} \\
\hline Astraptes fulgerator fulgerator (Walch, 1775) & & & $\mathrm{x}$ & & & & & & $\mathrm{x}$ & \\
\hline Aguna asander asander (Hewitson, 1867) & & & & & & & & $\mathrm{x}$ & $\mathrm{x}$ & \\
\hline Aguna metophis (Latreille, [1824]) & & & & $\mathrm{x}$ & & & & & $\mathrm{x}$ & \\
\hline Autochton neis (Geyer, 1832) & & & & $\mathrm{x}$ & $\mathrm{x}$ & & & & $\mathrm{x}$ & \\
\hline Chioides catillus catillus (Cramer, 1779) & & & $\mathrm{x}$ & & & & & & $\mathrm{x}$ & \\
\hline Epargyreus clavicornis clavicornis (Herrich-Schäffer, 1869) & & & $\mathrm{x}$ & & & & & & $\mathrm{x}$ & \\
\hline Euriphellus euribates (Stoll, 1782) & & & & $\mathrm{x}$ & & & & & $\mathrm{x}$ & \\
\hline Hyalothyrus leucomelas (Geyer, 1832) & & & $\mathrm{x}$ & & & & & & $\mathrm{x}$ & \\
\hline Phocides pigmalion hewitsonius (Mabille, 1883) & & & & $\mathrm{x}$ & & & & & $\mathrm{x}$ & \\
\hline
\end{tabular}

Phocides pigmalion hewitsonius (Mabille, 1883)

ia do Mearim; SBR = São Benedito do Rio Preto; SDA = São Domingos do Azeitão; EN $=$ Entomological net; $\mathrm{BT}=$ Baited trap. 
Table 1. Continued..

\begin{tabular}{|c|c|c|c|c|c|c|c|c|c|c|}
\hline \multirow{2}{*}{ Taxa (Family, Subfamily, Species, Subspecies) } & \multicolumn{6}{|c|}{ Amazon } & \multicolumn{2}{|c|}{ Cerrado } & \multicolumn{2}{|c|}{ Sampling method } \\
\hline & $\mathbf{C P}$ & PV & PM & SJR & SL & VT & SBR & SDA & EN & BT \\
\hline Typhedanus crameri McHenry, 1960 & $\mathrm{x}$ & & & & & & & & $\mathrm{x}$ & \\
\hline Udranomia orcinus (C. Felder \& R. Felder, 1867) & & & & $\mathrm{x}$ & & & & & $\mathrm{x}$ & \\
\hline Urbanus chalco (Hübner, 1823) & & & $\mathrm{x}$ & & & & & & $\mathrm{x}$ & \\
\hline Urbanus dorantes dorantes (Stoll, 1790) & & $\mathrm{x}$ & $\mathrm{x}$ & $\mathrm{x}$ & & $\mathrm{x}$ & $\mathrm{x}$ & & $\mathrm{x}$ & \\
\hline Urbanus procne (Plötz, 1881) & & $\mathrm{x}$ & $\mathrm{x}$ & & & & & & $\mathrm{x}$ & \\
\hline Urbanus proteus (Linnaeus, 1758) & $\mathrm{x}$ & & & $\mathrm{x}$ & & & $\mathrm{x}$ & & $\mathrm{x}$ & \\
\hline Urbanus simplicius (Stoll, 1790) & & $\mathrm{x}$ & $\mathrm{x}$ & $\mathrm{x}$ & & & & & $\mathrm{x}$ & \\
\hline \multicolumn{11}{|l|}{ Hesperiinae $(\mathrm{S}=\mathbf{2 3})$} \\
\hline Augiades crinisus (Cramer, 1780) & & & $\mathrm{x}$ & & & & & & $\mathrm{x}$ & \\
\hline Aides duma duma Evans, 1955 & & & $\mathrm{x}$ & & & & & & $\mathrm{x}$ & \\
\hline Aides duma argyrina Cowan, 1970 & & $\mathrm{x}$ & & & & & $\mathrm{x}$ & & $\mathrm{x}$ & \\
\hline Aides aegita (Hewitson, 1866) & & & & & $\mathrm{x}$ & & & & $\mathrm{x}$ & \\
\hline Calpodes ethlius (Stoll, 1782) & & & $\mathrm{x}$ & $\mathrm{x}$ & & & & & $\mathrm{x}$ & \\
\hline Carystoides basoches (Latreille, [1824]) & & & $\mathrm{x}$ & & & & & & $\mathrm{x}$ & \\
\hline Carystoides maroma (Möschler, 1877) & & & $\mathrm{x}$ & $\mathrm{x}$ & & & & & $\mathrm{x}$ & \\
\hline Carystus phorcus phorcus (Cramer, 1777) & & & $\mathrm{x}$ & & & & & & $\mathrm{x}$ & \\
\hline Cobalus calvina (Hewitson, 1866) & & & $\mathrm{x}$ & & & & & & $\mathrm{x}$ & \\
\hline Cymaenes tripunctus theogenis (Capronnier, 1874) & & & $\mathrm{x}$ & & & & & & $\mathrm{x}$ & \\
\hline Cynea irma (Möschler, 1879) & & $\mathrm{x}$ & & & & & & & $\mathrm{x}$ & \\
\hline Cynea robba robba Evans, 1955 & & & & $\mathrm{x}$ & & & & & $\mathrm{x}$ & \\
\hline Enosis uza uza (Hewitson, 1877) & & & $\mathrm{x}$ & & & & & & $\mathrm{x}$ & \\
\hline Mnasicles hicetaon Godman, 1901 & & & $\mathrm{x}$ & & & & & & $\mathrm{x}$ & \\
\hline Morys valerius (Möschler, 1879) & & & & & & & & $\mathrm{x}$ & $\mathrm{x}$ & \\
\hline Nyctelius nyctelius nyctelius (Latreille, [1824]) & & & $\mathrm{x}$ & & & & & & $\mathrm{x}$ & \\
\hline Panoquina fusina fusina (Hewitson, 1868) & & & & $\mathrm{x}$ & & & & & $\mathrm{x}$ & \\
\hline Phanes aletes (Geyer, 1832) & & & & & & & & $\mathrm{x}$ & $\mathrm{x}$ & \\
\hline Panoquina ocola ocola (Edwards, 1863) & & & $\mathrm{x}$ & & & & & & $\mathrm{x}$ & \\
\hline Polites vibex (Geyer, 1832) & & & & & $\mathrm{x}$ & & & & $\mathrm{x}$ & \\
\hline Pompeius pompeius (Latreille, [1824]) & & & $\mathrm{x}$ & & & & & & $\mathrm{x}$ & \\
\hline Pyrrhopygopsis socrates (Ménétriés, 1855) & $\mathrm{x}$ & & & & & & $\mathrm{x}$ & & $\mathrm{x}$ & \\
\hline Saliana saladin Evans, 1955 & & & & $\mathrm{x}$ & $\mathrm{x}$ & & & & $\mathrm{x}$ & \\
\hline Synale hylaspes (Stoll, 1781) & & & & & & & & $\mathrm{x}$ & $\mathrm{x}$ & \\
\hline \multicolumn{11}{|l|}{ Pyrginae $(S=14)$} \\
\hline Elbella sp Evans, 1951 & & & & $\mathrm{x}$ & & & & & $\mathrm{x}$ & \\
\hline Grais stigmaticus stigmaticus (Mabille, 1883) & & & $\mathrm{x}$ & & & & & & $\mathrm{x}$ & \\
\hline Heliopetes arsalte (Linnaeus, 1758) & $\mathrm{x}$ & $\mathrm{x}$ & $\mathrm{x}$ & $\mathrm{x}$ & & & & $\mathrm{x}$ & $\mathrm{x}$ & \\
\hline Heliopetes omrina (Butler, 1870) & $\mathrm{x}$ & & & & & $\mathrm{x}$ & & & $\mathrm{x}$ & \\
\hline Jemadia fallax fida Evans, 1951 & & & & $\mathrm{x}$ & & & & & $\mathrm{x}$ & \\
\hline Mysoria barcastus antila Evans, 1951 & & $\mathrm{x}$ & $\mathrm{x}$ & & & & & & $\mathrm{x}$ & \\
\hline Pyrgus orcus (Stoll, 1780) & & & $\mathrm{x}$ & $\mathrm{x}$ & & $\mathrm{x}$ & $\mathrm{x}$ & $\mathrm{x}$ & $\mathrm{x}$ & \\
\hline Pyrgus veturius Plötz, 1884 & & & & $\mathrm{x}$ & & & & & $\mathrm{x}$ & \\
\hline Pyrrhopyge phidias (Linnaeus, 1758) & & & & & & $\mathrm{x}$ & & & $\mathrm{x}$ & \\
\hline Pyrrhopyge sp Hübner, [1819] & & & $\mathrm{x}$ & & & & & & $\mathrm{x}$ & \\
\hline Timochares trifasciata (Hewitson, 1868) & & & & $\mathrm{x}$ & & & & & $\mathrm{x}$ & \\
\hline Timochreon satyrus (C. Felder \& R. Felder, 1867) & & & & & & & & $\mathrm{x}$ & $\mathrm{x}$ & \\
\hline Viola violella (Mabille, 1898) & & & & & & & $\mathrm{x}$ & & $\mathrm{x}$ & \\
\hline Zopyrion evenor evenor Godman, 1901 & & & & & & & & $\mathrm{x}$ & $\mathrm{x}$ & \\
\hline
\end{tabular}

CP = Cururupu; PV = Penalva; PM = Peri Mirim; SJR = São José de Ribamar; SL = São Luís; VT = Vitória do Mearim; SBR = São Benedito do Rio Preto; SDA = São Domingos do Azeitão; EN = Entomological net; BT = Baited trap.

Table 2. Butterfly inventories previously published in the state of Maranhão, Northeast Brazil.

\begin{tabular}{lccc}
\hline \multicolumn{1}{c}{ Authors and year } & Municipality & Coordinates & Sampling method \\
\hline Bates 1867 & Maracaçumé & $02^{\circ} 02^{\prime} \mathrm{S} 45^{\circ} 57^{\prime} \mathrm{W}$ & Entomological net \\
Garcia et al. 1990 & São Luís & $02^{\circ} 39^{\prime} \mathrm{S} 44^{\circ} 15^{\prime} \mathrm{W}$ & Entomological net \\
Garcia \& Bergman 1994 & São Luís & $02^{\circ} 39^{\prime} \mathrm{S} 44^{\circ} 15^{\prime} \mathrm{W}$ & Entomological net \\
Ramos 2000 & Açailândia & $5^{\circ} 01^{\prime} \mathrm{S}, 47^{\circ} 32^{\prime} \mathrm{W}$ & 39 \\
\hline
\end{tabular}

*There are 23 species mentioned in the paper, but the author states that 364 species were sampled and deposited in collections. 
well inventoried biomes regarding butterflies in Brazil (Santos et al. 2008), we observe higher richness in several sites of the later, which shelter from 218 to 914 species (Brown Jr \& Freitas 2000, Francini et al. 2011). On the other hand, the richness of 189 species that was recorded in the present study was greater than in three of the four butterfly lists previously performed in the state of Maranhão (Garcia et al. 1990, Garcia \& Bergman 1994, Ramos 2000), highlighting the need for greater sampling effort towards butterflies in the state. Although not directly comparable, these data indicate that new records should be expected in our less sampled study areas with the inclusion of rare and inconspicuous taxa, which are usually only captured after extensive samplings (Magurran 2004).

Patterns of endemism to a specific biome have been proposed for different groups of butterflies in Brazil (Pinheiro et al. 2010), suggesting that some of the collected species could be endemic. However, the task of determining endemism has been hampered by the scarcity of available data, especially for transition zones (Silva et al. 2005, Pinheiro et al. 2010). From the collected species, we highlight Nicolaea socia (Hewitson, 1868), which has been proposed to be endemic of Cerrado (Pinheiro et al. 2010). Considering that Nicolaea socia (Hewitson, 1868) was only captured in São Benedito do Rio Preto, we reinforce its distribution as an endemic species of this biome. The high number of species that only occurred in the Amazon (124) is probably related to the greater sampling effort applied in our Amazonian sites. Since Bates (1867), Garcia et al. (1990), Garcia \& Bergmann (1994) and Ramos (2000) field works were performed exclusively in the Amazon, we believe that new inventories in Maranhão's Cerrado are necessary to evaluate the distribution and endemism of butterflies from Maranhão.

The low similarity between species captured through entomological nets and cylindrical traps was expected. Entomological nets usually capture larger number of species, but its dependence on good weather and collector's abilities may limit the capture of inconspicuous groups (Sparrow et al. 1994). On the other hand, baited traps sample fewer species, but consistently yields species rarely observed by researchers (Sparrow et al. 1994, Freitas et al. 2014). Consequently, the low value of similarity obtained in this study reinforces that entomological nets and baited traps contribute with complementary data for butterfly inventories in the Neotropics (Sparrow et al. 1994, Caldas \& Robbins 2003).

\section{Taxonomic composition}

The richest family in our list was Nymphalidae, following the same pattern of other butterfly lists performed in Brazil (Zacca et al. 2011, Morais et al. 2012). Most recorded species of this family are widespread throughout the country, such as Hamadryas februa (Hübner, [1823]), Hamadryas feronia (Linnaeus, 1758), Hamadryas amphinome (Linnaeus, 1767), Junonia evarete (Cramer, 1779) and Agraulis vanillae (Linnaeus, 1758). The fact that Hamadryas februa (Hübner, [1823]) was our most widespread species is concerning but expected, since this species is characteristic of modified habitats and secondary forests (Brown Jr 1992). Individuals of Hamadryas februa (Hübner, [1823]) were also captured by Garcia et al. (1990), Garcia \& Bergmann (1994) and Ramos (2000), suggesting that this species is common in different regions of the state. Six species of the genus Heliconius Kluk, 1780, one of the most studied groups of butterflies regarding evolution and diversity patterns (Mallet 1993, Mavárez et al. 2006, Merrill et al. 2015), were sampled, including two races of Heliconius melpomene (Linnaeus, 1758): Heliconius melpomene melpomene (Linnaeus, 1758) and Heliconius melpomene nanna Stichel, 1899. Bates (1867) described different variations of Heliconius species captured in Maracaçumé, including intermediate varieties between Heliconius melpomene melpomene (Linnaeus, 1758) and Heliconius melpomene thelxiope Hübner [1806]. Considering the distribution of Heliconius butterflies and the suggested hybridization zones for this group in the Amazon (Mallet 1993, Merrill et al. 2015), new species and races of Heliconius are expected to be found in Maranhão.

The second richest family was Hesperiidae, including common species in the Neotropics, such as Astraptes fulgerator fulgerator (Walch, 1775), Autochton neis (Geyer, 1832) and Heliopetes arsalte (Linnaeus, 1758). Hesperiidae is considered, along with Nymphalidae, the richest families in the Neotropical region (Lamas 2004). Moreover, Francini et al. (2011) stated that Hesperiidae is expected to be the richest family in relatively complete inventories of Brazil, while Nymphalidae is the best represented butterfly group in short-term studies, probably because nymphalids are easily visualized and identified in the field. In this study, Nymphalidae was considerably richer than Hesperiidae, implying that additional records of hesperids should be expected with an increased sampling effort.

Riodinidae was the third family in species richness, followed by Lycaenidae. This result agrees with those from lists performed in other Amazonian sites (Emmel \& Austin 1990, Brown Jr \& Freitas 2002), although lists from other biomes frequently demonstrate Lycaenidae being richer than Riodinidae (Francini et al. 2011). Riodinidae richness has been correlated with the mean temperature of the study areas, explaining its greater number in tropical regions (Brown Jr 2005, Francini et al. 2011), such as Maranhão. From the captured riodinids, we highlight Napaea beltiana beltiana (Bates, 1867) and Napaea eucharila (Bates, 1867), species also captured and described by Bates (1867). Lycaenidae was the fourth family in number of species, but we expect the richness of Lycaenidae to increase, since this family include small and inconspicuous species, requiring longer temporal surveys for adequate samplings (Francini et al. 2011).

Pieridae and Papilionidae are the families with lowest species richness in Brazil (Emery et al. 2006, Francini et al. 2011), and our findings corroborate this pattern. Most captured species of Pieridae and Papilionidae are considered widely distributed throughout the country and characteristic of modified habitats (Emery et al. 2006, Zacca et al. 2011, Morais et al. 2012), suggesting that the study areas have been harmed by anthropic influences.

\section{Perspectives for future research}

Considering our sampling effort and the difficulties to sample a large Brazilian state, we estimate that the recorded species represent a small subset of the butterflies from Maranhão. Broader and longer samplings could result in much greater richness, especially for the families Hesperiidae, Riodinidae and Lycaenidae. Centuries of butterfly records from Maranhão in museum collections must also be analyzed in the future to achieve better understanding of butterflies' diversity and distribution in the state.

Two of the four lists previously performed in Maranhão were conducted at the state capital, São Luís. The proximity to the major research centers of the state and the easy access to study areas may be the main reason for this geographic bias. Consequently, little or no effort is applied for distant and complicated access areas, resulting in the current lack of information. Among the priority areas for butterfly inventories, the western region of Maranhão should be focused by researchers, since this portion of Amazon is still poorly studied and under human pressures (Silva et al. 2005, Martins \& Oliveira 2011). Moreover, this region is part of the most threatened endemism center in Brazil, the Belém Center of Endemism (Silva et al. 2005). Only about one-third of its forest remains, and the increasing deforestations may represent the extinction of several species, including endemic butterflies (Hall \& Harvey 2002, Silva et al. 2005). Therefore, inventories in this area are recommended to identify novel and/or endemic taxa. Long-term researches are also requested for Maranhão's Cerrado, since different butterfly species have been considered endemic of this endangered biome (Brown Jr \& Gifford 2002, Pinheiro et al. 2010) and there are no previous butterfly lists published for this region.

Species inventories are important tools for a better understanding of the biodiversity. In this context, it is urgent to perform studies that generate lists 
of species in poorly studied areas to evaluate the distribution and endemism of different taxa, such as megadiverse groups of insects. As previously mentioned, the richness of 189 butterfly species collected in the present study can be considered low when compared to intensively sampled areas in the Amazon and Cerrado. On the other hand, we presented the greatest number of species in butterfly inventories from Maranhão since Bates (1867). Furthermore, some of our study areas were sampled for the first time and may shelter a greater butterfly diversity, including species not yet known. Thus, we call attention to the urgent measures that should be taken to preserve Amazon and Cerrado remnants of Maranhão and their local biodiversity, since these areas have been increasingly threatened by anthropic disturbances.

\section{Acknowledgments}

We would like to thank the researchers of the Laboratório de Ecologia e Sistemática de Insetos Polinizadores e Predadores (LESPP/UFMA), especially David Barros Muniz for helping with the confection of the map. Marcelo Duarte helped in various phases of this project, including technical support. Renato de Oliveira, André Victor Lucci Freitas, Diego Rodrigo Dolibaina, Ricardo Russo Siewert and Jessie Pereira dos Santos helped greatly with identifications. Our sincere gratitude to Quercegen S.A and Instituto Formação for allowing us to develop part of this research in Sítio Aguahy and Parque Agroecológico de Buritirana, respectively. We are grateful to two anonymous reviewers for helpful comments on the original version of the manuscript. This study was supported by the Fundação de Amparo à Pesquisa e ao Desenvolvimento Científico e Tecnológico do Maranhão (FAPEMA/APP-UNIVERSAL-00404/11 and FAPEMA/ CBIOMA 02986/12).

\section{Author Contributions}

Lucas Pereira Martins: substantial contribution in the concept and design of the study; contribution to manuscript preparation, data collection, interpretation and analysis.

Elias da Costa Araujo Junior: substantial contribution in the concept and design of the study; contribution to manuscript preparation, data collection and critical revision.

Ananda Regina Pereira Martins: substantial contribution in the concept and design of the study; contribution to manuscript preparation and critical revision.

Mairla Santos Colins: substantial contribution to manuscript preparation, data collection and critical revision.

Gabriela Cristina Fonseca Almeida: substantial contribution to manuscript preparation, data collection and critical revision.

Gisele Garcia Azevedo: substantial contribution in the concept and design of the study; contribution to manuscript preparation and critical revision.

\section{Conflicts of interest}

The authors declare that they have no conflict of interest related to the publication of this manuscript.

\section{References}

BARRETO, L., VAN EUPEN, M., KOK, K., JONGMAN, R.H.G., RIBEIRO, M.C., VELDKAMP, A., HASS, A. \& OLIVEIRA, T.G. 2012. The impact of soybean expansion on mammal and bird, in the Balsas region, north Brasilian Cerrado. J Nat Conserv. 20(6):374-383.

BATES, H.W. 1867. On a collection of butterflies formed by Thomas Belt, Esq., in the interior of the province of Maranhão, Brazil. T Roy Ent Soc London. 5(7):535-546.
BONEBRAKE, T.C., PONISIO, L.C., BOGGS, C.L \& EHRLICH, P.R. 2010. More than just indicators: a review of tropical butterfly ecology and conservation. Biol. Conserv. 143(8):1831-41.

BROWN JR, K.S. 1992. Borboletas da Serra do Japi: diversidade, habitats, recursos alimentares e variação temporal. In Historia Natural da Serra do Japi: ecologia e preservação de uma área florestal no sudeste do Brasil (L. Morellato, ed). Unicamp, Campinas, p.142-187.

BROWN JR, K.S. 1996. Diversity of Brazilian Lepidoptera: History of study, methods for measurements, and use as indicators for genetic, specific and system richness. In Biodiversity in Brazil: a first approach (C.E.M. Bicudo \& N.A. Menezes, eds). CNPq/Instituto de Botânica, São Paulo, p.221-253.

BROWN JR, K.S. 1997. Diversity, disturbance, and sustainable use of Neotropical forests: insects as indicators for conservation monitoring. J Insect Conserv. $1(1): 25-42$.

BROWN JR, K.S. \& FREITAS, A.V.L. 2000. Atlantic forest butterflies: Indicators for Landscape Conservation. Biotropica 32(4b):934-956.

BROWN JR, K.S. \& FREITAS, A.V.L. 2002. Diversidade biológica no Alto Juruá: avaliação, causas e manutenção. In O Alto Juruá: Práticas e Conhecimentos das Populações (M.M.C. Cunha \& M.B. Almeida, eds). Enciclopédia da floresta, São Paulo, p.33-42.

BROWN JR, K.S. \& GIFFORD, D.R. 2002. Lepidoptera in the Cerrado landscape and the conservation of vegetation, soil, and topographical mosaics. In The Cerrados of Brazil: Ecology and Natural History of a Neotropical Savanna (P.S Oliveira \& R.J. Marquis, eds). Columbia University Press, New York, p.201-222.

BROWN JR, K.S. 2005. Geological, evolutionary and ecological bases of the diversification of Neotropical butterflies: implications for conservation. In Tropical rainforests: Past, Present and Future (E. Bermingham, C.W. Dick \& C., Moritz, eds). University of Chicago Press, Chicago, p.166-201.

CALDAS, A. \& ROBBINS, R.K. 2003. Modified Pollard transects for assessing tropical butterfly abundance and diversity. Biol. Conserv. 110:211-219.

CASAGRANDE, M.M., MIELKE, O.H.H., CARNEIRO, E., RAFAEL, J.A. \& R.W. HUTCHINGS. 2012. Hesperioidea e Papilionoidea (Lepidoptera) coligidos em expedição aos Rios Nhamundá e Abacaxis, Amazonas, Brasil: novos subsídios para o conhecimento da biodiversidade da Amazônia Brasileira. Rev Brasi Entomol 56(1):23-28.

DINIZ-FILHO, J.A.F., OLIVEIRA, G., LOBO, F., FERREIRA JR, L.G., BINI, L.M. \& RANGEL, T.F.V.L.B. 2009. Agriculture, habitat loss and spatial patterns of human occupation in a biodiversity hotspot. Sci Agric 66(6):764-771.

DUARTE, M., ROBBINS, R. K. 2010. Description and phylogenetic analysis of the Calycopidina (Lycaenidae, Theclinae, Eumaeini): a subtribe of detritivores. Rev Brasil Entomol. 54(1):45-65.

D'ABRERA, B. 1995. Butterflies of the Neotropical region: Nymphalidae. Hill House, Victoria.

EMERY, E.O., BROWN JR., K.S. \& PINHEIRO, C.E.G. 2006. As borboletas (Lepidoptera, Papilionoidea) do Distrito Federal, Brasil. Rev Brasil Entomol. 50(1):85-92.

EMMEL, T.C. \& AUSTIN, G.T. 1990. The tropical rain forest butterfly fauna of Rondônia, Brazil: species diversity and conservation. Trop Lepid. 1(1):1-12.

FRANCINI, R.B., DUARTE, M., MIELKE, O.H.H., CALDAS, A. \& FREITAS, A.V.L. 2011. Butterflies (Lepidoptera, Papilionoidea and Hesperioidea) of the "Baixada Santista" region, coastal São Paulo, southeastern Brazil. Rev Bras Entomol. 55(1):55-68

FREITAS, A.V., ISERHARD, C.A, SANTOS, J.P., CARREIRA, J.Y.O., RIBEIRO, D.B, MELO, D.H.A, ROSA, A.H.B, MARINI-FILHO, O.J., ACCACIO, G.M \& UEHARA-PRADO, M. 2014. Studies with butterfly bait traps: an overview. Rev Colomb Entomol. 40(2):209-218.

GARCIA, I.P., BERGMANN, E.C. \& NETTO, S.M.R. 1990. Diversidade mensal de borboletas da ilha de São Luís (MA). Arq. Inst. Biol. 57(1/2):39-44.

GARCIA, I.P. \& BERGMANN, E.C. 1994. Borboletas da Ilha de São Luís (MA) Arq. Inst. Biol. 56(1/2):37-38.

GARWOOD, K., LEHMAN, R., CARTER, W. \& CARTER, G. 2009. Butterflies of Southern Amazonia. A photographic checklist of common species. RiCalé Publishing, Texas. 
HALL, J.P.W. \& HARVEY, D. 2002. The phylogeography of Amazonia revisited: new evidence from riodinid butterflies. Evolution 56(7):1489-1497.

JOST, L., CHAO, A. \& CHAZDON R.L. 2011. Compositional similarity and beta diversity. In Biological Diversity: Frontiers in Measurement and Assessment (A.E. Magurran \& B.J. McGill, eds). Oxford University Press, Oxford, p.66-84.

KIM, K.C. 1993. Biodiversity, conservation, and inventory: why insects matter. Biodivers. Conser. 2(3):191- 214.

KLINK, C.A. \& MACHADO, R.B. 2005. Conservation of the Brazilian Cerrado. Conserv Biol. 19(3):707-713.

KREMEN, A.C., COLWELL, R.K., ERWIN, T.L., MURPHY, D.D., NOSS, R.F. \& SANJAYAN, M.A. 1993. Terrestrial Arthropod Assemblages: Their Use in Conservation Planning. Conserv Biol. 7(4):796-808.

LAMAS, G. 2004. Checklist: Part 4A. Hesperioidea - Papilionoidea. In Atlas of Neotropical Lepidoptera. Association for Tropical Lepidoptera (J. B. Heppner, ed). Scientific Publishers, Gainesville, p.1- 439.

LAURANCE, W.F., VASCONCELOS, H. L. \& LOVEJOY, T. E. 2000. Forest loss and fragmentation in the Amazon: implications for wildlife conservation. Oryx. 34(1):39-45.

LEWINSOHN, T.M., FREITAS, A.V.L. \& PRADO, P.I. 2005. Conservation of Terrestrial Invertebrates and Their Habitats in Brazil. Conserv Biol. 19(3):640-645.

MAGURRAN, A.E. 2004. Measuring biological diversity, 1 ed. Blackwell, Oxford.

MALLET, J. 1993. Speciation, raciation and color pattern evolution in Heliconius butterflies: evidence from hybrid zones. In Hybrid Zones and the Evolutionary Process (R.G. Harrison, ed.) Oxford University Press, New York, p.226-260.

MARTINS, M.B \& OLIVEIRA, T.G. 2011. Amazônia Maranhense: Diversidade e Conservação. 1 ed. MPEG, Belém.

MAVÁREZ, J., SALAZAR, C.A., BERMINGHAM, E., SALCEDO, C., JIGGINS, C.D. \& LINARES, M. 2006. Speciation by hybridization in Heliconius butterflies. Nature. 441:868-871.

MERRILL, R.M., DASMAHAPATRA, K.K., DAVEY, J.W., DELL'AGLIO, D.D., HANLY, J.J., HUBER, B., JIGGINS, C.D., JORON, M., KOZAK, K.M., LLAURENS, V., MARTIN, S.H., MONTGOMERY, S.H., MORRIS, J., NADEAU, N.J., PINHARANDA, A.L., ROSSER, N., THOMPSON, M.J., VANJARI, S., WALLBANK, R.W.R. \& YU, Q. 2015. The diversification of Heliconius butterflies: what have we learned in 150 years? J. Evol. Biol. 28:1417-1438.

MIELKE, O.H.H. 2005. Catalogue of the American Hesperioidea: Hesperiidae (Lepidoptera). 6 ed. Sociedade Brasileira de Zoologia, Curitiba.

MIELKE, O.H.H., CARNEIRO, E. \& CASAGRANDE, M.M. 2010. Lepidopterofauna (Papilionoidea e Hesperioidea) do Parque Estadual do Chandless e arredores, Acre. Biot Neotrop. 10(4): http://www.biotaneotropica.org.br/v10n4/pt/ fullpaper?bn03210042010 (last access on 27/05/2017)

MORAIS, A.B.B., LEMES, R. \& RITTER, C.D. 2012. Borboletas (Lepidoptera: Hesperioidea e Papilionoidea) de Val de Serra, região central do Rio Grande do Sul, Brasil. Biot Neotrop. 12(2): http://www.biotaneotropica.org.br/v12n2/ en/fullpaper?bn01412022012 (last access on 01/02/2017)

MYERS, N., MITTERMEIER, R.A., MITTERMEIER, C.G., DA FONSECA, G.A.B. \& KENT, J. 2000. Biodiversity hotspots for conservation priorities. Nature. 403:853-858.

PINHEIRO, C.E.G., MALINOV, I.K., EMERY, E.O. \& SCHMIDT, K. 2010. Endemismos e conservação de borboletas (Lepidoptera: Papilionoidea e Hesperioidea) no bioma Cerrado. In Cerrado: Conhecimento científico quantitativo como subsídio para ações de conservação (I.R. Diniz, J. Marinho-Filho, R.B. Machado \& R.B. Cavalcanti, eds). UNB, Brasília, p.225-238.

RAMOS, F.A. 2000. Nymphalid butterfly communities in an Amazonian forest fragment. J Res Lepid. 35:29-41.

ROBBINS R.K., LAMAS, G., MIELKE, O.H., HARVEY, D.J., CASAGRANDE, M.M. 1996. Taxonomic composition and ecological structure of the speciesrich butterfly community at Pakitza, Parque Nacional del Manu, Peru. In La Biodiversidad del Sureste del Peru. Lima, Peru (D.E. Wilson \& A. Sandoval, eds.). Smithsonian Institution Press, Washington D.C., p.201-236.

SANTOS, E.C, MIELKE, O.H.H. \& CASAGRANDE, M.M. 2008. Butterfly inventories in Brazil: the state of the art and the priority-areas model for research aiming at conservation. Nat Conservação. 6(2):178-200.

SANTOS, J.P., MARINI-FILHO, O.J., FREITAS, A.V.L. \& UEHARA-PRADO, M. 2016. Monitoramento de Borboletas: o Papel de um Indicador Biológico na Gestão de Unidades de Conservação. Biodiversidade Brasileira. 6(1):87-99.

SILVA, J. M. C., RYLANDS, A. B. \& FONSECA, G. A. B. 2005. O destino das áreas de endemismo da Amazônia. Megadiversidade. 1(1):124-131.

SPARROW, H.R., SISK, T.D., EHRLICH, P.R. \& MURPHY D.D. 1994. Techniques and guidelines for monitoring neotropical butterflies. Conserv Biol. 8(3):800-809.

VIEIRA, I.C.G., TOLEDO, P.M., SILVA, J.M.C. \& HIGUCHI, H. 2008. Deforestations and threats to the biodiversity of Amazonia. Braz J Biol. 68(4):949-956.

UEHARA-PRADO, M., BROWN JR., K.S. \& FREITAS, A.V.L. 2007. Species richness, composition and abundance of fruit-feeding butterflies in the Brazilian Atlantic Forest: comparison between a fragmented and a continuous landscape. Glob Ecol Biogeog. 16(1):43-54.

ZACCA, T., BRAVO, F., ARAÚJO, M.X. 2011. Butterflies (Lepidoptera: Papilionoidea and Hesperioidea) from Serra da Jibóia, Bahia State, Brazil. Entomobr. 4(3):139-143.
Received: $20 / 02 / 2017$

Revised: 28/05/2017

Accepted: 13/08/2017

Published online: 28/08/2017 